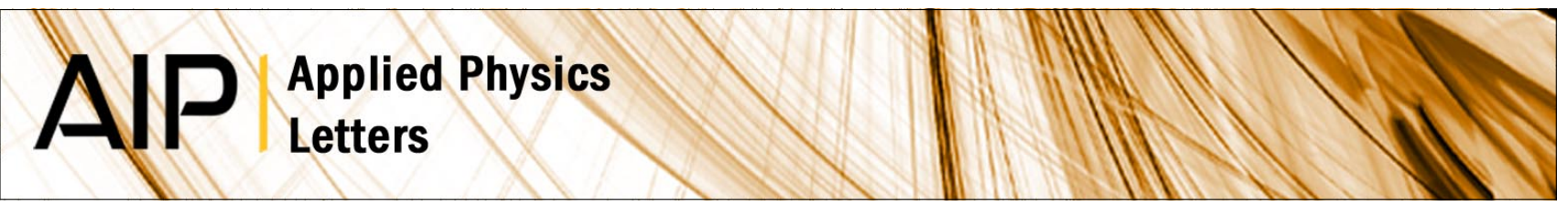

\title{
Understanding the role played by Fe on the tuning of magnetocaloric effect in Tb5Si2Ge2
}

A. M. Pereira, A. M. dos Santos, C. Magen, J. B. Sousa, P. A. Algarabel et al.

Citation: Appl. Phys. Lett. 98, 122501 (2011); doi: 10.1063/1.3567920

View online: http://dx.doi.org/10.1063/1.3567920

View Table of Contents: http://apl.aip.org/resource/1/APPLAB/v98/i12

Published by the American Institute of Physics.

\section{Related Articles}

High performance magnetocaloric perovskites for magnetic refrigeration Appl. Phys. Lett. 100, 121905 (2012)

Effect of microstrain on the magnetism and magnetocaloric properties of MnAs0.97P0.03 Appl. Phys. Lett. 100, 112407 (2012)

The magnetic and magnetocaloric properties of NdFe12-xMox compounds

J. Appl. Phys. 111, 07 A949 (2012)

Behavior of magnetic entropy change in perovskite Ba2-xLaxFeMoO6

J. Appl. Phys. 111, 07A947 (2012)

Magnetic and calorimetric studies of magnetocaloric effect in La0.7-xPrxCa0.3MnO3

J. Appl. Phys. 111, 07D726 (2012)

\section{Additional information on Appl. Phys. Lett.}

Journal Homepage: http://apl.aip.org/

Journal Information: http://apl.aip.org/about/about_the_journal

Top downloads: http://apl.aip.org/features/most_downloaded

Information for Authors: http://apl.aip.org/authors

\section{ADVERTISEMENT}

NEW!

iPeerReview

AIP's Newest App

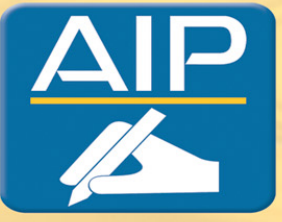

Authors...

Reviewers...

Check the status of

submitted papers remotely!

AIP Publishing 


\title{
Understanding the role played by Fe on the tuning of magnetocaloric effect in $\mathrm{Tb}_{5} \mathrm{Si}_{2} \mathrm{Ge}_{2}$
}

\author{
A. M. Pereira, ${ }^{1, a)}$ A. M. dos Santos, ${ }^{2}$ C. Magen, ${ }^{3,4}$ J. B. Sousa, ${ }^{1}$ P. A. Algarabel, ${ }^{4,5}$ \\ Y. Ren, ${ }^{6}$ C. Ritter, ${ }^{7}$ L. Morellon, ${ }^{4,5,8}$ M. R. Ibarra, ${ }^{4,5,8}$ and J. P. Araújo ${ }^{1}$ \\ ${ }^{1}$ IFIMUP and IN-Institute of Nanoscience and Nanotechnology, Departamento de Fisica de Faculdade de \\ Ciencias da Universidade do Porto, Rua do Campo Alegre, 687, 4169-007 Porto, Portugal \\ ${ }^{2}$ NSSD, P.O. Box 2008, MS6475, Oak Ridge, Tennessee 37831-6475, USA \\ ${ }^{3}$ Instituto de Nanociencia de Aragón-ARAID, Universidad de Zaragoza, 50009 Zaragoza, Spain \\ ${ }_{5}^{4}$ Departamento de Física de la Materia Condensada, Universidad de Zaragoza, 50009 Zaragoza, Spain \\ ${ }^{5}$ Instituto de Ciencia de Materiales de Aragón, Universidad de Zaragoza and Consejo Superior de \\ Investigaciones Científicas, 50009 Zaragoza, Spain \\ ${ }^{6}$ Advanced Photon Source, Argonne National Laboratory, Argonne, Illinois 60439, USA \\ ${ }^{7}$ Institut Laue-Langevin, Boîte Postale 156, 38042 Grenoble Cedex 9, France \\ ${ }^{8}$ Instituto de Nanociencia de Aragón, Universidad de Zaragoza, 50009 Zaragoza, Spain
}

(Received 7 February 2011; accepted 18 February 2011; published online 21 March 2011)

\begin{abstract}
In this letter, it is shown that when replacing Ge by $\mathrm{Fe}$ in $\mathrm{Tb}_{5} \mathrm{Si}_{2} \mathrm{Ge}_{2}$ the structural transition still occurs and enhances the magnetocaloric effect (MCE) (up to 66\%) with maximum of MCE at a critical Fe amount where the magnetic and structural transitions become fully coupled. It is observed that $\mathrm{Fe}$ concentration is able to mimic the effect of external pressure as it induces a complex microstructure, that tunes long range strain fields. This knowledge is crucial for the development of strategies toward materials with improved performance for efficient magnetic refrigeration applications. (C) 2011 American Institute of Physics. [doi:10.1063/1.3567920]
\end{abstract}

Refrigeration technologies are essential for modern society, especially those which are highly efficient and environmental friendly. ${ }^{1-3}$ Motivated by the potential for magnetic refrigeration applications at room temperature, the tuning of the properties of $\mathrm{Gd}_{5} \mathrm{Si}_{2} \mathrm{Ge}_{2}$ (GSG) is being exploited to increase its critical temperature while simultaneously maintaining or improving the magnitude of the giant magnetocaloric effect (MCE). The first work toward this goal was performed by Pecharsky and Gschneider in $1997,{ }^{2}$ by studying the effect of alloying many $3 d$-metal and $p$-element in GSG, where it was observed a reduction in the MCE, indicating changes in the thermodynamic nature of the magnetic phase transition. Afterwards, it was found that substitution of Ge by $\mathrm{Pb}$ and $\mathrm{Sn}$ in $\mathrm{Gd}_{5} \mathrm{Si}_{2} \mathrm{Ge}_{2}$ enhances the MCE by $\sim 11 \%$ leading, however, to only a slight increase in the critical temperature $\left(T_{\max }=274\right.$ and $281 \mathrm{~K}$, respectively). ${ }^{4,5}$ In the course of this research, a large improvement was obtained for compounds with a small amount of Ge (5 at. \%) replaced by $\mathrm{Fe}$. Here a drastic reduction in the hysteretic losses, an increase in the critical temperature and an improvement of the magnetic refrigeration efficiency were observed. ${ }^{6}$ However, a decrease in the MCE value was observed and attributed to a possible suppression of the structural phase transition. ${ }^{6}$ Similar results were obtained through the incorporation of other chemical elements (namely, first row transition metals) in the $\mathrm{Si} / \mathrm{Ge}$ network of GSG-based compounds, leading to similar results. ${ }^{5}$ Nevertheless, the physical origin of these effects is not clear. Unlike the case of the $\mathrm{Gd}_{5}\left(\mathrm{Si}_{x} \mathrm{Ge}_{1-x}\right)_{4}$ system, where the monoclinic (M) crystallographic phase is always found in the paramagnetic state, the $\mathrm{Tb}_{5} \mathrm{Si}_{2} \mathrm{Ge}_{2}$ compound presents a $\mathrm{M}$ structure which is already in the ferromagnetic state ${ }^{7}$ also presenting a considerable MCE. ${ }^{8}$ The slight decoupling of the magnetic and structural

${ }^{a)}$ Electronic mail: ampereira@fc.up.pt. transitions $\left[T_{S}=100 \mathrm{~K}\right.$ and $T_{C}=110 \mathrm{~K}$ (on cooling) $]$ (Ref. 7) offers a unique scenario ideal to study the impact of Fe replacement on the structural and magnetic transitions, being this is the main objective of the work presented herein.

The polycrystalline samples here studied were synthesized by the traditional arc-melting method. Further details about preparation can be found elsewhere. ${ }^{9}$ The microstructure studies were performed by scanning electron microscopy (SEM) and energy-dispersive x-ray spectroscopy (EDS). Low-field dc magnetization measurements and linear thermal expansion (LTE) were carried out simultaneously, in a modified commercial (Quantum Design) superconducting quantum interference device (SQUID) magnetometer. The high resolution $\mathrm{x}$-ray diffraction experiments were performed on the 11-D-C instrument $(\lambda=0.1066 \AA)$, at the Advanced Photon Source at Argonne National Laboratory (USA) in the temperature range of $10-250 \mathrm{~K}$. In support information, the neutron diffraction (ND) experiments were carried out on the high intensity powder diffractometer D1B $(\lambda=2.52 \AA)$ at the Institute Laue-Langevin in Grenoble (France). The crystallographic characterization of the samples was made by $\mathrm{Ri}-$ etveld refinement of the x-ray synchrotron diffraction (XRS) and ND patterns using the FULLPROF software package. ${ }^{10}$

Magnetization measurements show that the systematic substitution of Ge by $\mathrm{Fe}$ in $\mathrm{Tb}_{5} \mathrm{Si}_{2} \mathrm{Ge}_{2-y} \mathrm{Fe}_{y}$ leads to an increase in $T_{C}$ [Fig. 1(a)]; measured on heating from 112 to $123 \mathrm{~K}$ for $\mathrm{y}=0$ and 0.20 , respectively. A similar increase is observed on $T_{S}$ indicating the existence of the structural phase transition as observed by LTE and XRS data [Figs. 1(b) and 2]. The LTE measurements show a considerable change in the $\Delta L / L$ at $T_{S}$ [see Fig. 1(b)]. In the case of XRS data, Rietveld refinements indicate a complete conversion of the $\mathrm{O}(\mathrm{I})$ phase to $\mathrm{M}$ phase (on heating). This can be followed in Figs. 2(a) and 2(b), namely, through the appearance upon heating of the characteristic reflections of the $M$ phase $[(-1$ 

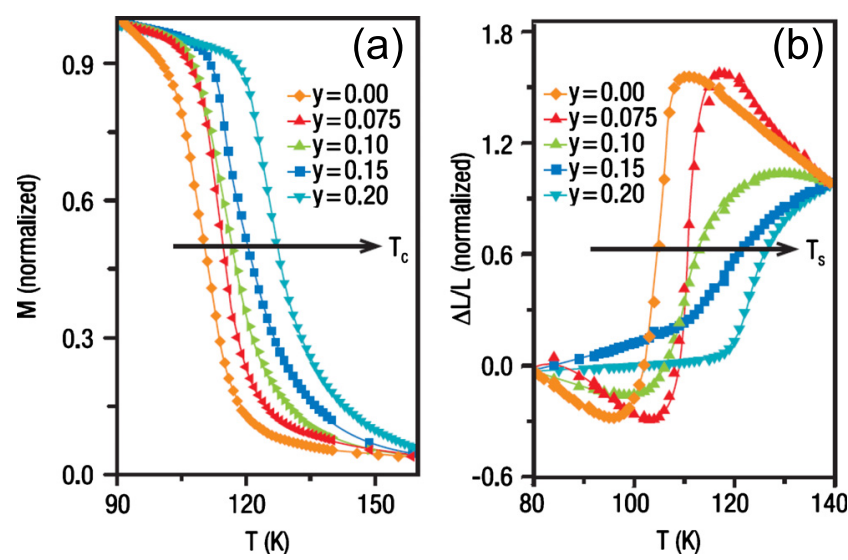

FIG. 1. (Color online) (a) Temperature dependence of the magnetization for $\mathrm{Tb}_{5} \mathrm{Si}_{2} \mathrm{Ge}_{2-y} \mathrm{Fe}_{y}$ compounds with $\mathrm{y}=0,0.075,0.10,0.15$ and 0.20 normalized at $\mathrm{M}(\mathrm{T}) / \mathrm{M}(\mathrm{T}=70 \mathrm{~K})$ for $H_{a p}=50$ Oe. (b) Temperature dependence of LTE normalized as $\frac{\Delta L}{L}=[\operatorname{LTE}(T)-\operatorname{LTE}(T=80 \mathrm{~K})] /[\operatorname{LTE}(T$ $=140 \mathrm{~K})-\operatorname{LTE}(T=80 \mathrm{~K})]$.

3 2), ( 042 ), and (-1 4 2)] and through the disappearance of the characteristic ones of the $\mathrm{O}(\mathrm{I})$ phase $\left[\begin{array}{lll}1 & 3 & 2\end{array}\right),\left(\begin{array}{lll}1 & 5 & 1\end{array}\right)$ and (2 4 1)]. A large thermal hysteresis is observed in LTE and XRS data corresponding to the coexistence of the $\mathrm{M}$ and $\mathrm{O}(\mathrm{I})$ phases (bright zone in Fig. 2) in accordance with the results reported in Ref. 7. It is noteworthy that the increase in the Fe concentration also leads to an increase in the reflection at about $2.41^{\circ}$. This corresponds to the $\left(\begin{array}{lll}2 & 1 & 1\end{array}\right)$ reflection of the $\mathrm{Tb}_{5}(\mathrm{Si}, \mathrm{Ge})_{3}$ secondary phase (5:3 phase; $\mathrm{Mn}_{5} \mathrm{Si}_{3}$-type). The presence of this impurity phase is further sustained by SEM and EDS analysis of the samples, displayed in Fig. 2, showing typical Widmanstaetten-type plates (linear microstructure) already for low $\mathrm{Fe}$ concentration $[\mathrm{y}=0.05$, Fig. 2(a)] (Refs. 11 and 12) with the amount of these plates increasing with the Fe content. The EDS results reveal a systematic absence of $\mathrm{Fe}$ in the main phase (5:4), meaning that iron is primarily accumulated in the secondary $5: 3$ phase and also incorporated in $\mathrm{Tb}(\mathrm{Si}, \mathrm{Ge}) \mathrm{Fe}$ (1:1:1) (found in our samples in trace amounts). In addition, EDS results indicate that the 5:4 phase is systematically enriched with Ge (meaning that silicon is preferentially located on the precipitates of the secondary phases). This is in agreement with the Rietveld refinements, that reveal an slightly increase in the unit cell volume of the 5:4 phase upon the increase in the Fe content. From the same refinements one also obtains a linear increase

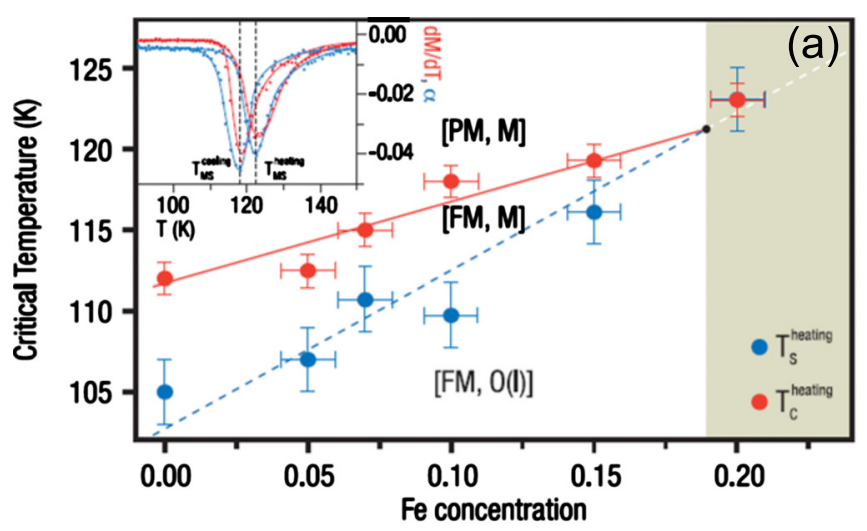

(a) $y=0$

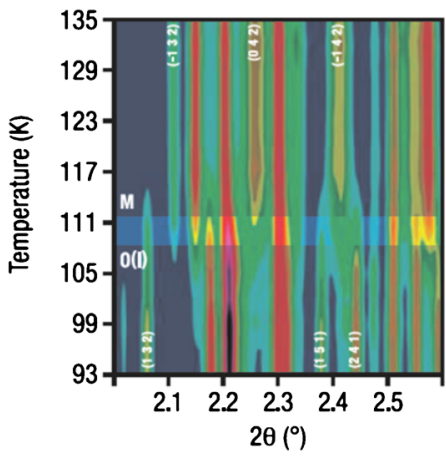

(c) $y=0.05$ (b) $y=0.20$

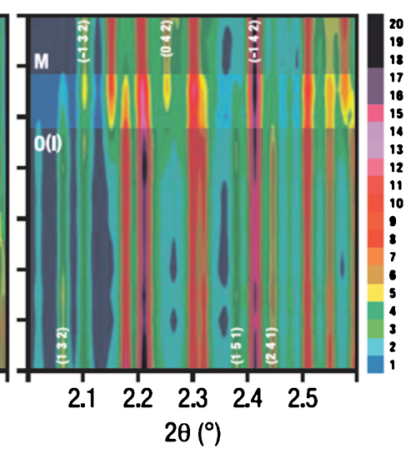

$y=0.15$

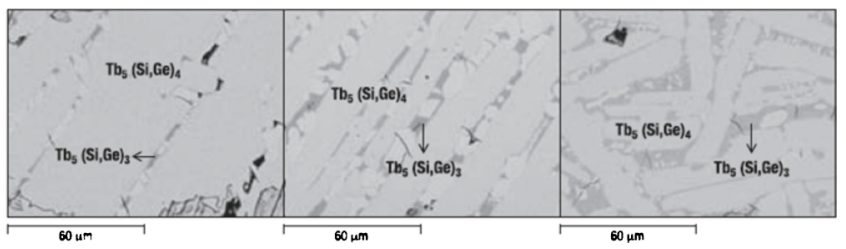

FIG. 2. (Color online) Temperature dependence of XRS data with $\lambda$ $=0.1066 \AA$, in the $93-135 \mathrm{~K}$ temperature range (on heating) for (a) $\mathrm{y}=0$ and (b) $y=0.20$. (c) backscattered electron SEM micrographs of samples with $\mathrm{y}=0.05,0.075$ and 0.15 .

in the percentage of the $5: 3$ phase with a maximum of $23 \%$ for the $y=0.20$ composition. The amount of $1: 1: 1$ phase can be considered negligible ( $<1 \%$ amount). The magnetostructural $(y, T)$ phase diagram is shown in Fig. 3(a), where the values of the transition temperatures were determined from the maxima of the derivative of the thermal dependence of the corresponding macroscopic properties. Both $T_{S}$ and $T_{C}$ shift linearly with $\mathrm{Fe}$ concentration to higher temperatures (Fig. 3). Notice that $\mathrm{d} T_{S} /$ dy is steeper than $\mathrm{d} T_{C} /$ dy, revealing that the substitution by $\mathrm{Fe}$ is directly influencing the structure. Above $\mathrm{y} \sim 0.19$, a fully coupled magneticcrystallographic transformation $[\mathrm{M}, \mathrm{PM}] \rightarrow[\mathrm{O}(\mathrm{I}), \mathrm{FM}]$ takes place as can be seen in the inset of Fig. 3(a), which displays the simultaneous occurrence of the magnetic and structural transition (through the $\mathrm{dLTE} / \mathrm{dT}$ and $\mathrm{dM} / \mathrm{dT}$ curves). This result is reinforced by ND experiments performed on compound with $y=0.15$ (see Ref. 13).

Figure 3(b) presents the temperature dependence of the magnetic entropy change $\left(\Delta S_{m}\right)$, obtained from the traditional Maxwell relation, on the Fe content for a magnetic

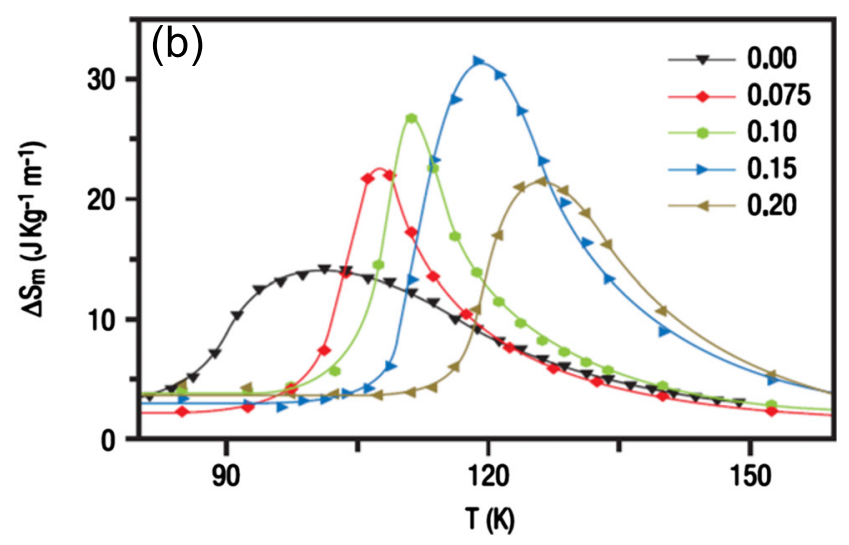

FIG. 3. (Color online) (a) Magnetostructural phase diagram of $\mathrm{Tb}_{5} \mathrm{Si}_{2} \mathrm{Ge}_{2-y} \mathrm{Fe}_{y}$ determined from magnetization and LTE data (for clarity only the values upon heating are displayed). Inset: $\mathrm{dM} / \mathrm{dT}$ and $\mathrm{dLTE} / \mathrm{dT}$. (b) MCE for $\mathrm{H}=50 \mathrm{kOe}$. 
field variation of $50 \mathrm{kOe}$ (no significant difference is observed in $\Delta S_{m}$ values when the magnetic field is increasing or decreasing). ${ }^{14} \mathrm{~A}$ drastic enhancement of the MCE up to $y=0.15$ is observed. The observed values of the MCE results the sum of the conventional magnetic entropy-driven process arising from the magnetic transition, $\Delta S_{\text {mag }}$, plus the difference in magnetic entropy of the two crystallographic structures, $\Delta S_{s t}{ }^{15}$ At higher concentration a fully coupled magnetic and structural transition occurs with a decrease in the magnetic entropy.

There is an intriguing parallelism between the effect of the substitution of Ge by $\mathrm{Fe}$ and the application of an external pressure on $\mathrm{Tb}_{5} \mathrm{Si}_{2} \mathrm{Ge}_{2}$, previously reported by Morellonet al. ${ }^{15}$ In that Letter, it was shown that for a sufficiently high external pressure, a full coupling between the magnetic and structural transitions occurred in a tricritical point leading to the corresponding enhancement of $\Delta S$, in accordance with our present results. Unfortunately no data of the MCE was obtained well above the tricritical point in this pressure study. However, the decrease in the MCE with the application of an external pressure was already observed in the fully coupled magnetostructural transition of $\mathrm{Gd}_{5} \mathrm{Si}_{2} \mathrm{Ge}_{2}$ (similarly to the present case after the fully coupled transitions) and in the MnAs. ${ }^{16,17}$

The features found here seem to be directly related to the complexity of the microstructure observed in the samples strongly influencing the first order magnetostructural transition (FOMT). The presence of quenched thin plates of 5:3 phase on the FOMT of $R_{5}\left(\mathrm{Si}_{x} \mathrm{Ge}_{1-x}\right)_{4}$ compounds has been the subject of several studies, ${ }^{11,12,18,19}$ and it was found that an "epitaxial" interface with an angle of $7^{\circ}$ between the $(10$ $0)$ and $\left(\begin{array}{llll}0 & 0 & 0 & 1\end{array}\right)$ reflections of the $5: 4$ phase and of the $5: 3$ plates, respectively, was created. This gives rise to longrange strain fields in the grain boundaries (on the interface) providing a similar effect as external pressure. The aforementioned mechanism has been suggested in Ref. 20 to explain the first-order $[\mathrm{PM}, \mathrm{M}] \rightarrow[\mathrm{FM}, \mathrm{O}(\mathrm{I})]$ transition in $\mathrm{Gd}_{5} \mathrm{Ge}_{2} \mathrm{Si}_{2}$ single crystal, where it was inferred that longrange strain fields, arising from complex platelet/matrix interface $[(5: 3) /(5: 4)$ phases, respectively], are the source of strain. They also showed that this persistent local strain promotes the $\mathrm{PM} \rightarrow \mathrm{FM}$ transition but not the inverse. ${ }^{20}$ In conclusion, the discovery that $\mathrm{Fe}$ substitution in $R_{5}\left(\mathrm{Si}_{x} \mathrm{Ge}_{1-x}\right)_{4}$ compounds operates similarly to external pressure, reinforcing the magnetostructural coupling via internal strain, opens wide perspectives for materials engineered with optimized properties for magnetic refrigeration applications.

Work partially supported by the projects FEDER/POCTI $\mathrm{n}^{0} 155 / 94, \mathrm{POCI} / \mathrm{CTM} / 61284 / 2004$ and PTDC/CTM/NAN/
115125/2009 from Fundação para a Ciência e Tecnologia (FCT), Portugal. A.M.P. thanks FCT for the Grant No. SFRH/BPD/63150/2009. The financial support of the Spanish MEC (Grant No. MAT2008-06567-C02) and DGA (Grant No. E26) is acknowledged. Use of the Advanced Photon Source at Argonne National Laboratory was supported by the U.S. Department of Energy, Office of Science, Office of Basic Energy Sciences, under Contract No. DE-AC0206CH11357.

${ }^{1}$ A. de Campos, D. L. Rocco, A. M. G. Carvalho, L. Caron, A. A. Coelho, S. Gama, L. M. Da Silva, F. C. G. Gandra, A. O. Dos Santos, L. P. Cardoso, P. J. von Ranke, and N. A. de Oliveira, Nature Mater. 5, 802 (2006) .

${ }^{2}$ V. Pecharsky and K. A. Gschneidner, Jr., Phys. Rev. Lett. 78, 4494 (1997).

${ }^{3}$ V. Pecharsky and K. Gschneidner, Jr., J. Magn. Magn. Mater. 167, L179 (1997).

${ }^{4}$ Y. Zhuang, J. Li, W. Huang, W. Sun, and W. Ao, J. Alloys Compd. 421, 49 (2006).

${ }^{5}$ R. Shull, V. Provenzano, A. Shapiro, A. Fu, M. Lufaso, J. Karapetrova, G. Kletetschka, and V. Mikula, J. Appl. Phys. 99, $08 k 908$ (2006).

${ }^{6}$ V. Provenzano, A. Shapiro, and R. Shull, Nature (London) 429, 853 (2004).

${ }^{7}$ L. Morellon, C. Ritter, C. Magen, P. Algarabel, and M. R. Ibarra, Phys. Rev. B 68, 024417 (2003).

${ }^{8}$ L. Morellon, C. Magen, P. Algarabel, M. R. Ibarra, and C. Ritter, Appl. Phys. Lett. 79, 1318 (2001).

${ }^{9}$ A. M. Pereira, J. B. Sousa, J. P. Araujo, C. Magen, P. A. Algarabel, L. Morellon, C. Marquina, and M. R. Ibarra, Phys. Rev. B 77, 134404 (2008).

${ }^{10}$ J. Rodriguez-Carvajal, Physica B 192, 55 (1993).

${ }^{11}$ L. Chumbley, O. Ugurlu, R. McCallum, K. Dennis, Y. Mudryk, K. A Gschneidner, Jr., and V. K. A. Pecharsky, Acta Mater. 56, 527 (2008).

${ }^{12}$ J. Meyers, S. Chumbley, F. Laabs, and A. Pecharsky, Acta Mater. 51, 61 (2003).

${ }^{13}$ See supplementary material at http://dx.doi.org/10.1063/1.3567920 for Temperature dependence of ND showing the fully coupling of the magnetic and structural transitions.

${ }^{14}$ J. Amaral and V. Amaral, Appl. Phys. Lett. 94, 042506 (2009).

${ }^{15}$ L. Morellon, Z. Arnold, C. Magen, C. Ritter, O. Prokhnenko, Y. Skorokhod, P. Algarabel, M. R. Ibarra, and J. Kamarad, Phys. Rev. Lett. 93, 137201 (2004).

${ }^{16}$ A. M. G. Carvalho, C. S. Alves, A. de Campos, A. A. Coelho, S. Gama, F. C. G. Gandra, P. J. von Ranke, and N. A. Oliveira J. Appl. Phys. 97, 10M320 (2005).

${ }^{17}$ S. Gama, A. A. Coelho, A. deCampos, A. M. Carvalho, F. C. G. Gandra, P. J. Von Ranke, and N. A. De Oliveira, Phys. Rev. Lett. 93, 237202 (2004)

${ }^{18}$ O. Ugurlu, L. Chumbley, D. Schlagel, and T. Lograsso, Acta Mater. 54, 1211 (2006)

${ }^{19}$ Z. W. Ouyang, V. K. Pecharsky, K. A. Gschneidner, Jr., and T. A. Lograsso, Phys. Rev. B 74, 024401 (2006).

${ }^{20}$ J. D. Moore, K. Morrison, G. K. Perkins, D. L. Schlagel, T. A. Lograsso, and K. A. G. Jr, V. K. Pecharsky, and L. F. Cohen, Adv. Mater. 21, 1 (2009). 